\title{
A Thermo-elastic Annular Plate Model for the Modeling of Brake Systems
}

\author{
José Luis Reyes Pérez*, Andreas Heckmann* and Ingo Kaiser* \\ * German Aerospace Center (DLR), Institute of Robotics and Mechatronics \\ Oberpfaffenhofen, 82234 Wessling, Germany
}

\begin{abstract}
The friction forces generated during braking between brake pads and discs produce high thermal gradients on the rubbing surfaces. These thermal gradients may cause braking problems such as hot spotting and the associated hot judder phenomenon in the frequency range below $100 \mathrm{~Hz}$.

Some consequences of these undesirable vibrations are comfort reductions, a defective braking process, inhomogeneous wear, cutbacks of the brake performance and even damage of brake components.

The present paper proposes a modeling concept that is targeted on this field of application and introduces the new Modelica class ThermoelasticPlate, which is implemented in the DLR FlexibleBodies library.

Keywords: Disc brake, Modal multifield approach, Thermoelasticity
\end{abstract}

\section{Introduction}

Friction braking is necessarily related to high thermal loads which lead to high thermal gradients at the surface of brake discs. It is a known phenomenon that these thermal loads can initiate the onset of unevenly distributed hot spots or bands which in turn results in thermally deformed brake discs [1], [2]. Since the brake pads then slide upon a non-smooth surface while the brake disc rotates, the brake system vibrates, noise is generated and undesirable wear occurs.

Besides experimental studies the finite element method (FEM) [3], [4], [5] [6] or analytical techniques [7] [8] are utilized to analyze the thermo-elastic behavior of brakes in literature. Both methods have advantages and provide valuable results, but both methods are not well suited, if complex scenarios such as the interaction of brakes with suspensions or vehicle control systems are investigated and a system dynamical point of view is adopted.
To this purpose the present paper proposes a novel model of a moderately simplified brake disc. Depending on the user input the thermo-elastic behavior of brake discs is described with approximately 100 up to 1000 degrees of freedom.

The thermal field of the disc is discretized in three dimensions in Eulerian representation, an annular Kirchhoff plate is adapted to evaluate the deformations according to the quasi-static thermo-elastic theory $[9$, Ch. 2].

In circumferential direction the disc is assumed to be rotational symmetric, in axial direction different layers with different heat capacity and conduction properties and multiple surfaces, where cooling by convection occurs, may be defined.

In order to implement this concept the Modelica class ThermoelasticPlate has been introduced into the commercial DLR FlexibleBodies library. This paper presents the underlying theory on thermal and thermoelastic fields, explains the user interface of the ThermoelasticPlate class and gives an simulation example. The final section gives an outlook to further efforts in research and modeling of friction brakes and its validation, which is supposed to be initiated by the novel modeling approach.

\section{Thermal Field}

\subsection{Weak Field Equations}

In order to describe the thermal behaviour of the brake disc the weak equations for the temperature field $\vartheta(\boldsymbol{c}, t)$ as functions of the spatial position in cylindrical coordinates $c=(r, \phi, z)^{T}$ and time $t$ are deduced from the principle of virtual temperature, see e.g. [10, (7.7)] or [11, (1.3.33)]: 


$$
\begin{aligned}
\int_{V}\left[-(\nabla \delta \vartheta)^{T} \boldsymbol{q}+\rho c \dot{\vartheta} \delta \vartheta\right] \mathrm{d} V+\ldots \\
\ldots+\int_{B} \boldsymbol{q}_{B}^{T} \boldsymbol{n}_{B} \delta \vartheta \mathrm{d} B=0,
\end{aligned}
$$

where $\rho$ denotes the density, $c$ the specific heat capacity, $\mathrm{d} B$ the boundary element and $\boldsymbol{n}_{B}$ the outer unit normal vector. $\boldsymbol{q}$ symbolizes the heat flux according to Fourier's law of heat conduction depending on the temperature gradient $\nabla \vartheta$ and the thermal conductivity matrix $\boldsymbol{\Lambda}[9,(1.12 .16)]$ :

$$
q=-\Lambda \nabla \vartheta
$$

The boundary heat flux $q_{B}$ may be given explicitly or, if convection occurs, may be specified by the film coefficient $h_{f}$ and the bulk temperature $\vartheta_{\infty}$ of the fluid [9, Sec. 5.6]:

$$
\boldsymbol{q}_{B}^{T} \boldsymbol{n}_{B}=-q_{B}-h_{f}\left(\vartheta_{B}-\vartheta_{\infty}\right)
$$

\subsection{Modal Approach}

The discretization of the scalar temperature is performed using the Ritz approximation that allows to separate the thermal field description by a finitedimensional linear combination of two parts, the first one considers thermal modes and is spatial dependent, i.e. $\boldsymbol{\Phi}_{\vartheta}=\boldsymbol{\Phi}_{\vartheta}(\boldsymbol{c})$ and the second one represent the modal amplitudes and is time dependent, i.e. $\boldsymbol{z}_{\vartheta}=\boldsymbol{z}_{\vartheta}(t)$ :

$$
\vartheta(\boldsymbol{c}, t)=\boldsymbol{\Phi}_{\vartheta}(\boldsymbol{c}) \boldsymbol{z}_{\vartheta}(t)
$$

The spatial mode functions are formulated using the separation approach of Bernoulli for the spatial coordinates as well, so that (4) may be rewritten as follows:

$$
\begin{aligned}
& \sum_{i=1}^{n} \Phi_{\vartheta i}(\boldsymbol{c}) z_{\vartheta i}(t)=\sum_{i=1}^{n} R_{i}(r) \Psi_{i}(\phi) Z_{i}(z) z_{\vartheta i}(t)= \\
& =\sum_{l=1}^{l_{m}} \sum_{k=0}^{k_{m}} \sum_{m=1}^{m_{m}} R_{l}(r) \cdot \cos (k \phi) \cdot Z_{m}(z) \cdot z_{\vartheta i}(t)+ \\
& \quad+\sum_{l=1}^{l_{m}} \sum_{k=1}^{k_{m}} \sum_{m=1}^{m_{m}} R_{l}(r) \cdot \sin (k \phi) \cdot Z_{m}(z) \cdot z_{\vartheta i}(t),
\end{aligned}
$$

with $i=1,2, \ldots, n, n=\left(l_{m} m_{m}\right)\left(2 k_{m}+1\right)$.

According to Walter Ritz [12], the trial functions have to be linearly independent and components of a complete system, so that the number of $i$ may be increased as needed in order to improve the approximation. For $R_{l}(r)$ and $Z_{m}(z)$ cubic B-splines [13] as

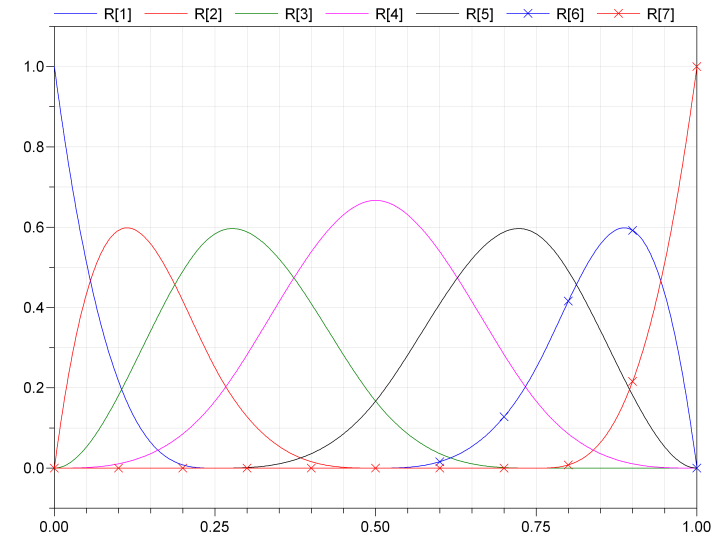

Figure 1: Example set of cubic B-splines to discretize the thermal field in radial and axial direction.

shown in Fig. 1 have been chosen as trial functions in radial and axial direction, respectively.

The harmonic waves (or Fourier series expansion) $\Psi_{i}(\phi)$ are appropriate in circumferential direction, since

- they allow to represent cyclic properties, i.e. $\Psi_{i}(\phi)=\Psi_{i}(\phi+2 \pi)$

- they are simple to integrate from 0 to $2 \pi$,

- their orthogonality leads to block-diagonal system matrices, i.e. the entire system of equations is split up into decoupled sub-systems,

- they will later on be exploited to provide a Eulerian description of the thermo-elastic plate.

\subsection{Discretized Field Equations}

If (5) is inserted into (1), the volume integrals can be separated from the terms that dependent on time. As a result, the linear thermal field equation is obtained:

$$
\boldsymbol{C}_{\vartheta \vartheta} \dot{\boldsymbol{z}}_{\vartheta}+\left(\boldsymbol{K}_{\vartheta \vartheta}+\boldsymbol{K}_{\vartheta R}\right) \boldsymbol{z}_{\vartheta}=\boldsymbol{Q}_{\vartheta N} q_{B}+\boldsymbol{Q}_{\vartheta R} \vartheta_{\infty}
$$

where the volume integrals are defined, inter alia using the abbreviation $\boldsymbol{B}_{\vartheta}:=\nabla \boldsymbol{\Phi}_{\vartheta}$, as follows [14, Tab. 2.5]:

the heat capacity matrix:

$$
C_{\vartheta \vartheta}:=\int_{V} \rho c \boldsymbol{\Phi}_{\vartheta}^{T} \boldsymbol{\Phi}_{\vartheta} \mathrm{d} V
$$

the conductivity matrix:

$\boldsymbol{K}_{\vartheta \vartheta}:=\int_{V} \boldsymbol{B}_{\vartheta}^{T} \boldsymbol{\Lambda} \boldsymbol{B}_{\vartheta} \mathrm{d} V$

the Robin load matrix:

$\boldsymbol{K}_{\vartheta R}:=\int_{B} h_{f} \boldsymbol{\Phi}_{\vartheta}^{T} \boldsymbol{\Phi}_{\vartheta} \mathrm{d} B$

the Robin load vector:

$\boldsymbol{Q}_{\vartheta R}:=\int_{B} h_{f} \boldsymbol{\Phi}_{\vartheta}^{T} \mathrm{~d} B$

the Neumann load vector:

$Q_{\vartheta N}:=\int_{B} \Phi_{\vartheta}^{T} \mathrm{~d} B$ 
These volume integrals may therefore be evaluated in advance to the simulation or time integration, respectively.

\subsection{The Eulerian Description}

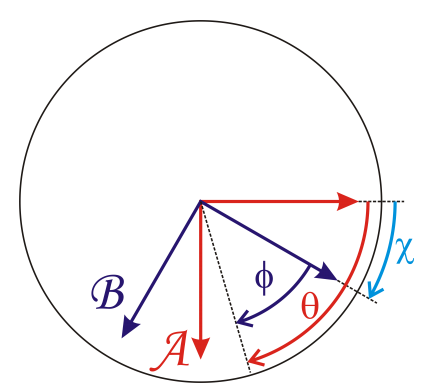

Figure 2: Coordinate transformation with angle $\chi$, that leads from the Lagrangian to the Eulerian description.

It is now considered that the brake disc performs a rotation around its central axis specified by the angle $\chi(t)$. So far the temperature field is described in the so-called Lagrangian point of view [15, Sec. I.3], i.e. the reference frame follows the rotation as it is shown for the coordinate system named $\mathcal{B}$ in Fig. 2.

However for the specific use case treated here it may make sense to resolve the temperature field of the disc in frame $\mathcal{A}$ in Fig. 2. In other words, the observer does not rotate with the disc but looks on the plate from the outside, from a point in rest concerning the rotation with angle $\chi(t)$.

This concept is the so-called Eulerian description [15, Sec. I.4] and is widely used in fluid dynamics, where the motion state of the fluid at a fixed point in space is presented. Due to the rotational symmetry properties of the brake disc the Eulerian description can here be formulated in an elegant and convenient way.

For theoretical derivation the coordinate transformation

$$
\phi=\theta-\chi
$$

is defined, where $\theta$ specifies the angular position of an observed point on the brake disc resolved with respect to the Eulerian reference system $\mathcal{A}$ in Fig. 2.

Furthermore it is assumed that for every trial function in (5) that employs a $\sin (k \phi)$-term an associated trial function is present where the sinus- is replaced by the cosinus-function only, but $R_{l}(r), Z_{m}(z)$ and $k$ are identical, so that mode shape couples $c_{1}$ and $c_{2}$ exist:

$$
\begin{aligned}
& c_{1}(r, \phi, z)=R_{l}(r) \cdot Z_{m}(z) \cdot \sin (k \phi), \\
& c_{2}(r, \phi, z)=R_{l}(r) \cdot Z_{m}(z) \cdot \cos (k \phi) .
\end{aligned}
$$

If the following identities

$$
\begin{aligned}
\sin (k \phi) & =\sin (k \theta) \cos (k \chi)-\cos (k \theta) \sin (k \chi), \\
\cos (k \phi) & =\cos (k \theta) \cos (k \chi)+\sin (k \theta) \sin (k \chi)
\end{aligned}
$$

are inserted into (8), an associated mode couple $\bar{c}_{1}(r, \theta, z)$ and $\bar{c}_{2}(r, \theta, z)$ defined with respect to frame $\mathcal{A}$ appears:

$$
\begin{aligned}
& c_{1}=\underbrace{R_{l} Z_{m} \sin (k \theta)}_{:=\bar{c}_{1}(r, \theta, z)} \cos (k \chi)- \\
&-\underbrace{R_{l} Z_{m} \cos (k \theta)}_{:=\bar{c}_{2}(r, \theta, z)} \sin (k \chi), \\
& c_{2}=\bar{c}_{1}(r, \theta, z) \sin (k \chi)+\bar{c}_{2}(r, \theta, z) \cos (k \chi) .
\end{aligned}
$$

As a result of suitable transformations it may also be written:

$$
\begin{aligned}
& \bar{c}_{1}(r, \theta, z)=c_{2} \sin (k \chi)+c_{1} \cos (k \chi), \\
& \bar{c}_{2}(r, \theta, z)=c_{2} \cos (k \chi)-c_{1} \sin (k \chi) .
\end{aligned}
$$

The mode functions $\bar{c}_{1}(r, \theta, z)$ and $\bar{c}_{2}(r, \theta, z)$ are defined in the Eulerian reference system $\mathcal{A}$ and are linear combinations of the mode functions $c_{1}(r, \phi, z)$ and $c_{2}(r, \phi, z)$ described in the Lagrangian frame $\mathcal{B}$, whereas the combination depends on $\chi(t)$.

This information can be exploited in order to define a transformation: a thermal field resolved in the Lagrangian frame can be transformed to be resolved in the Eulerian frame and vice versa. Of course the physical temperature field itself does not change, but its resolution does so that the numerical values describing the field will be different in frame $\mathcal{A}$ or $\mathcal{B}$, respectively.

In practice the transformation is formulated in terms of the modal amplitudes $z_{\vartheta i}(t)$ which are the thermal states in (6):

$$
\begin{aligned}
& \bar{z}_{\vartheta i 1}(t)=\sin (k \chi(t)) z_{\vartheta i 2}(t)+\cos (k \chi(t)) z_{\vartheta i 1}(t), \\
& \bar{z}_{\vartheta i 2}(t)=\cos (k \chi(t)) z_{\vartheta i 2}(t)-\sin (k \chi(t)) z_{\vartheta i 1}(t) .
\end{aligned}
$$

Again, the new modal amplitudes in the Eulerian frame $\bar{z}_{\vartheta i}(t)$ are expressed as a linear combination of modal amplitudes in the Lagrangian frame $z_{\vartheta i}(t)$ and it is just a matter of convenience and practicability in which coordinates the thermal field equations are actually evaluated.

One particularity has been ignored so far. For trial functions with $k=0$ no mode couple with $c_{1}$ and $c_{2}$ according to (8) exists, since no associated sinusfunction is introduced in (5). As a consequence the transformation (12) is not defined for such modes. However, trial functions with $k=0$ represent rotational symmetric fields since the dependency on $\phi$ is 
eliminated in (5) due to the term $\cos (k \phi)$. As a consequence mode shapes with $k=0$ are invariant with respect to rotations with angle $\chi$ or in other words: The modal coordinates $z_{\vartheta i}(t)$ related to $k=0$ are identical in the Eulerian and the Lagrangian description and no transformation is needed.

\section{Mechanical Field}

The present paper is focused on the thermo-elastic interrelation that rules the behavior of brake discs in frequency range below $100 \mathrm{~Hz}$. Note that there is a complementary paper presented on this Modelica User Conference which is dedicated to higher frequencies in order to cope e.g. with brake squeal phenomena [16]. However here, it is supposed that the excitation is much lower than the lowest natural frequency of the brake disc. In particular the following assumption are made:

- The structural deformations of the brake disc are dominated by its elasticity or thermo-elasticity, respectively, while inertia effects are negligible. The brake disc deforms in a quasi-static manner. This statement is related to the so-called Duhamel's assumption which argues on the different time-scales with which changes in the temperature or deformation field usaully proceed, $\mathrm{cp}$. [9, Sec. 2.5].

- A literature review on the characteristics of thermo-elastic brake disc deformation give reason to the assumption that plate bending in some cases even plate buckling is the governing deformation mechanism, see [7], [1], [4]. For example: all experimental studies describe e.g. hot spots to be located alternatively on the two disc surfaces in anti-symmetrical configuration, so that the circumference is deformed similar to a sinuous line. Therefore the deformation field of the brake disc here is represented as an annular Kirchhoff plate.

Note that the description of the annular plate is limited to be linear in this initial implementation, so that plate buckling phenomena are not covered, see [17], [18, Ch. 1]. An extended formulation to consider thermal buckling is a field of active research at the DLR.

\subsection{Thermo-elastic Coupling}

In [14, Sec. 2.2] the material constitution based on a thermodynamical potential is harnessed to formulate the interrelation of the thermal and the mechanical field. This approach is not suited here, since the influence of a 3-dimensional thermal on a 2-dimensional displacement field is to describe.

Instead the so-called body-force analogy is employed, i.e. the thermoelastic problem is transfered into an isothermal problem with equivalent distributed body forces $\boldsymbol{\sigma}_{\vartheta}[9, \S 3.3]$, whose non-zeros components in radial and tangential direction read:

$$
\sigma_{\vartheta r}=\sigma_{\vartheta \phi}=-\frac{1+v}{1-v^{2}} E \alpha \vartheta
$$

where $\alpha$ denotes the thermal expansion coefficient, $E$ Young's modulus and $v$ the Poisson number. Together with the relevant strain components in radial and tangential direction $\varepsilon_{r}$ and $\varepsilon_{\phi}$ expressed as functions of the transversal plate deformation $w$

$$
\varepsilon_{r}=-z w_{, r r}, \quad \varepsilon_{\phi}=-z\left(\frac{w_{, r}}{r}+\frac{w_{, \phi \phi}}{r^{2}}\right)
$$

the associated virtual work $\delta W_{\vartheta}$ reads:

$$
\begin{aligned}
& \delta W_{\vartheta}=\int_{V} \delta \varepsilon^{T} \sigma_{\vartheta} \mathrm{d} V= \\
& =E \alpha \frac{1+v}{1-v^{2}} \int_{V} \delta\left(\begin{array}{c}
w_{, r r} \\
\frac{w_{, r}}{r}+\frac{w_{, \phi \phi}}{r^{2}}
\end{array}\right)^{T}\left(\begin{array}{c}
z \vartheta \\
z \vartheta
\end{array}\right) \mathrm{d} V
\end{aligned}
$$

\subsection{Weak Field Equations}

The structural displacements $\boldsymbol{u}$ are evaluated on the basis of the principle of virtual displacements [10, (4.7)], which states that the virtual work of the internal forces equals the virtual work of the external forces:

$$
\int_{V} \delta \varepsilon^{T} \boldsymbol{\sigma} \mathrm{d} V+\int_{V} \delta \varepsilon^{T} \boldsymbol{\sigma}_{\vartheta} \mathrm{d} V=\sum_{i} \delta \boldsymbol{u}^{T} \boldsymbol{f}_{i},
$$

where $\varepsilon$ denotes the strain and $\sigma$ the stress field. $f_{i}$ represent the applied external forces.

\subsection{Modal Approach}

Again a Ritz approximation is used to discretize the deformation field $\boldsymbol{u}$ :

$$
\boldsymbol{u}(\boldsymbol{c}, t)=\boldsymbol{\Phi}_{u}(\boldsymbol{c}) \boldsymbol{z}_{u}(t)
$$

The spatial shape functions in (17) are formulated as function of cylindrical coordinates, i.e. $\boldsymbol{\Phi}_{u}=$ 
$\boldsymbol{\Phi}_{u}(r, \phi, z), w_{, r}$ and $w_{, \phi}$ are partial derivatives with respect to $r$ or $\phi$ :

$$
\begin{gathered}
\sum_{i=1}^{n} \boldsymbol{\Phi}_{u i} z_{u i}=\left[\begin{array}{c}
-z\left(\cos (\phi) w_{, r}-\frac{\sin (\phi)}{r} w_{, \phi}\right) \\
-z\left(\sin (\phi) w_{, r}+\frac{\cos (\phi)}{r} w_{, \phi}\right) \\
w
\end{array}\right] \\
w=\sum_{l=0}^{l_{m}} \sum_{k=0}^{k_{m}} R_{l}(r) \cdot \cos (k \phi) \cdot z_{u i}(t)+\ldots \\
\ldots+\sum_{l=0}^{l_{m}} \sum_{k=1}^{k_{m}} R_{l}(r) \cdot \sin (k \phi) \cdot z_{u i}(t)
\end{gathered}
$$

with $i=1,2, \ldots, n, n=\left(l_{m}+1\right)\left(2 k_{m}+1\right)$.

The trial functions in (18) correspond to the trial functions in (5) except of the fact, that a 2-dimensional field is discretized here, while the temperatures depend on all three coordinates.

\subsection{Discretized Field Equations}

If (18) is inserted into (16) the linear field equation for the displacements is yielded:

$$
\boldsymbol{K}_{u u} \boldsymbol{z}_{u}+\boldsymbol{K}_{u \vartheta} \boldsymbol{z}_{\vartheta}=\sum_{i} \boldsymbol{\Phi}_{u i}^{T} \boldsymbol{f}_{i}
$$

The stiffness matrix $\boldsymbol{K}_{u и}$ in (19) is defined using the linear displacement-strain operator $\nabla_{u}$, the abbreviation $\boldsymbol{B}_{u}:=\nabla_{u} \boldsymbol{\Phi}_{u}$ and the elasticity tensor $\boldsymbol{H}$ :

$$
\boldsymbol{K}_{u u}:=\int_{V} \boldsymbol{B}_{u}^{T} \boldsymbol{H} \boldsymbol{B}_{u} \mathrm{~d} V
$$

The thermo-elastic coupling matrix follows from (15):

$$
\begin{aligned}
\boldsymbol{K}_{u \vartheta}:= & E \alpha \frac{1+v}{1-v^{2}} \ldots \\
& \ldots \int_{V}\left(\boldsymbol{\Phi}_{u, r r}+\frac{\boldsymbol{\Phi}_{u, r}}{r}+\frac{\boldsymbol{\Phi}_{u, \phi \phi}}{r^{2}}\right) z \boldsymbol{\Phi}_{\vartheta} \mathrm{d} V
\end{aligned}
$$

In addition to the deformations, the motion of the disc's reference frame located at the center of gravity is considered by the Newton-Euler equations [19, (8.6),(8.21)]:

$$
\begin{aligned}
m \cdot a & =\sum f_{i} \\
I \dot{\omega}+\omega \times I \boldsymbol{\omega} & =\sum c_{i} \times f_{i}+\sum p_{i} .
\end{aligned}
$$

$\boldsymbol{a}$ denotes the translational acceleration of the reference frame, $\boldsymbol{\omega}$ its rotational velocity. $\boldsymbol{I}$ symbolizes the interia tensor, $m$ its mass. $\boldsymbol{f}_{i}$ presents the discrete external forces, $\boldsymbol{p}_{i}$ discrete external torques.

\section{User Interface}

The user interface in Dymola is shown in Fig. 3: here the BrakeForce module represents the input force applied on the brake pads, the PAD module defines the specific locations of the ALE nodes where the one end of the springs will be attached to and provide the kinematics which are fed into the CONTACT module. The CONTACT module is the set of springs and dampers which conects the brake pads with the brake disc.

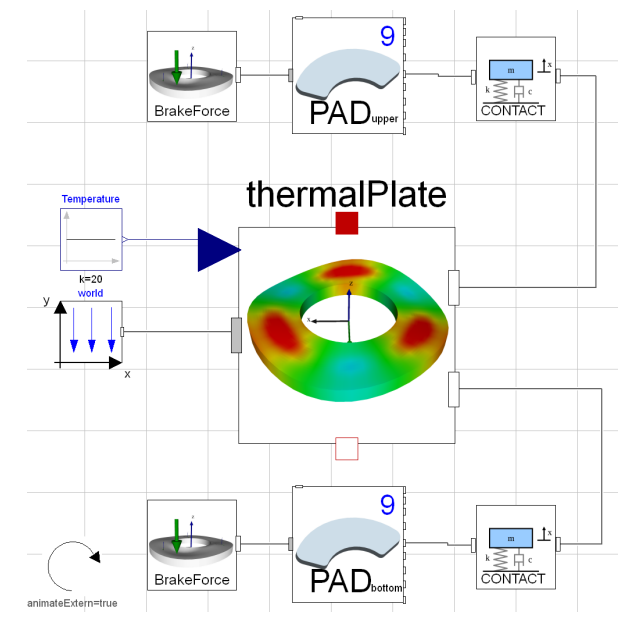

Figure 3: User interface of the thermo-elastic plate.

Finally the thermalPlate module is the block which contains the thermo-mechanical description of the plate which has been derived in the previews sections and also includes the geometrical parameters to model the annular plate (in Table 1).

\begin{tabular}{|l|c|}
\hline PARAMETERS & DESCRIPTION \\
\hline \hline$r_{-} i$ & Inner radius [m] \\
$r_{-} a$ & Outer radius [m] \\
$t h$ & Thickness [m] \\
$\left.x_{s i} i:, 2\right]$ & Specific points on the plate \\
\hline
\end{tabular}

Table 1: Geometrical parameters of the plate .

The thermalPlate module contains two types of connectors: the frame of reference and two array frames which represent specific points distributed over the bottom and upper surface of the disc in ALE description. The connectors nodes_ALE_upper and nodes_ALE_bottom are defined by the array $x$ si. Each row of $x$ si defines the radial and angular position of one point over the parametrized disc surface contained in the interval $[0,1]$, i.e. if we have $x \operatorname{si}[1,:]=\{0.5$, $0.125\}$ the point will be localized in the middle of the distance between the outer radius and the inner radius at $45^{\circ}$ in angular position. 
In real applications brake discs are subjected to very high increments of temperature during braking which might have a negative impact on the braking performance. In order to reduce the influence of such temperature gradients the so called cooling channels are integrated in the structure allowing the air to flow through the mid part of the disc, providing a faster dissipation of the heat transferred to the brake disc. The impact of the cooling channels on the structural dynamics has been also taken into account when modeling the thermo-elastic plate in a simplified way. The idea was to divide the plate thickness in 3 regions (Fig. 4) where the outer regions have a different heat transfer coefficient than the inner region. The calculation of the heat transfer coefficients is not trivial; therefore some assumptions had been done.

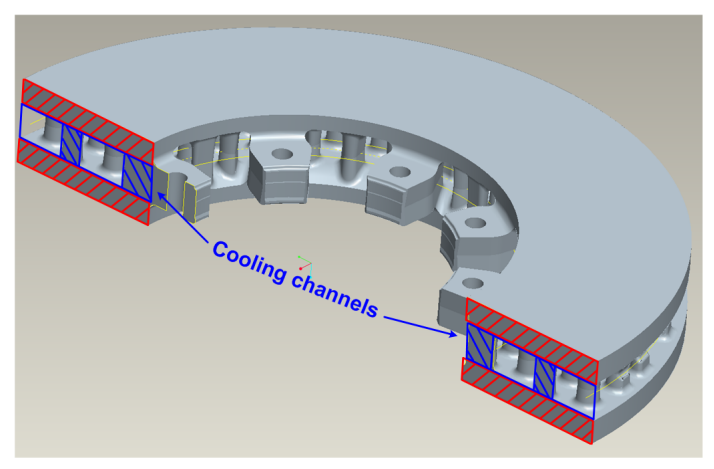

Figure 4: Cooling channels in a brake disc.

Note that conventional frame connectors from the Standard Multibody Library are used within the FlexibleBodies Library, so there is no restriction by connecting other bodies or elements to the nodes_ALE connectors. The thermalPlate module offers the possibility to select the initial conditions to which the annular plate is exerted and also the discretization parameters that control the modal approach of the annular plate:

- boundaryConditionRI: this parameter specifies the boundary condition at the inner radius and provides the options free, supported and clamped.

- boundaryConditionRA: this parameter specifies the boundary condition at the outer radius and provides the options free, supported and clamped.

- radialDiscretization: this is an integer vector of arbitrary length, in which all nodal diameters numbers to consider have to be given.

- angularlDiscretization: this is an integer vector of arbitrary length, in which all nodal circles to consider have to be given.
The model has been implemented in the Standard FlexibleBody Library as a complementary example to the already known Beam and ModalBody classes.

\section{Simulation Example}

The following example is a simplified representation of a braking system which illustrates an application of the thermo-elastic plate model. The mechanism consists of two brake pads and a thermo-elastic plate. The pads can only perform translations in the direction of the $\mathrm{z}$-axis whereas the thermo-elastic plate rotates around the z-axis with a constant angular velocity. The geometrical, mechanical and thermal properties of the thermo-elastic plate are listed in Table 2.

\begin{tabular}{|l|c|}
\hline PlATE DESCRIPTION & VALUE \\
\hline \hline Inner radius [m] & 0.075 \\
Outer radius [m] & 0.15 \\
Thickness [m] & 0.022 \\
\hline Density $\left[\mathrm{kg} / \mathrm{m}^{3}\right.$ ] & 7850 \\
Thermal conductivity [W/m.K] & 47 \\
Specific heat [J/kg.K] & 70 \\
Thermal expansion coefficient [1/K] & $1.04 \mathrm{e}-5$ \\
\hline Young's Modulus [Pa] & $2.1 \mathrm{e} 11$ \\
Poisson's Ratio & 0.29 \\
\hline
\end{tabular}

Table 2: Properties and dimensions of the plate .

During this simulation a force, called normal force, is acting on both brake pads along the axial direction. According to Figure 5 this force is set to zero at the beginning and after certain time step (100 s) the force is increased up to $5 \mathrm{kN}$. Once this force is applied, the brake pads will tend to move enforcing the contact with the surfaces of the disc and thus the increment of the temperature in the disc due to friction.

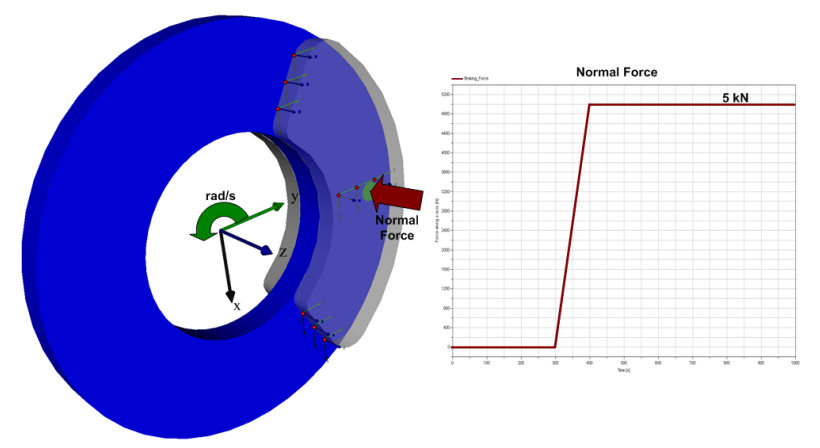

Figure 5: Sketch of the plate with the ALE nodes and normal force.

The contact model consists of a system of dis- 
crete linear springs and dampers located at 9 specific nodes on the pads and assembled with its corresponding nodes on the disc. The nodes on the disc are selected according to the ALE formulation (red dots in Fig. 5) and they are fixed in space so that they do not rotate with the disc. At these ALE nodes the forces generated by the springs and dampers are applied to the disc. Due to the sliding friction, heat is generated and it is induced into the brake disc.

Figures 6 and 7 contain the displacement in zdirection and the applied forces of the selected 9 ALE nodes respectively. It is important to observe how the deformation is increased as well as the forces at the ALE nodes as a result of not only the external forces (e.g. normal force) but also internal forces (e.g. thermal stresses) acting in the brake disc.

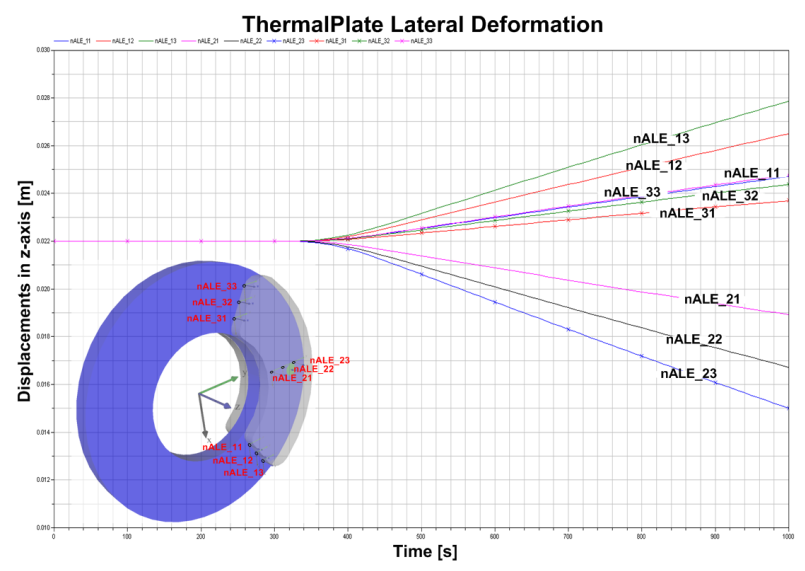

Figure 6: Results of the thermalPlate model for the deformation along z-axis.

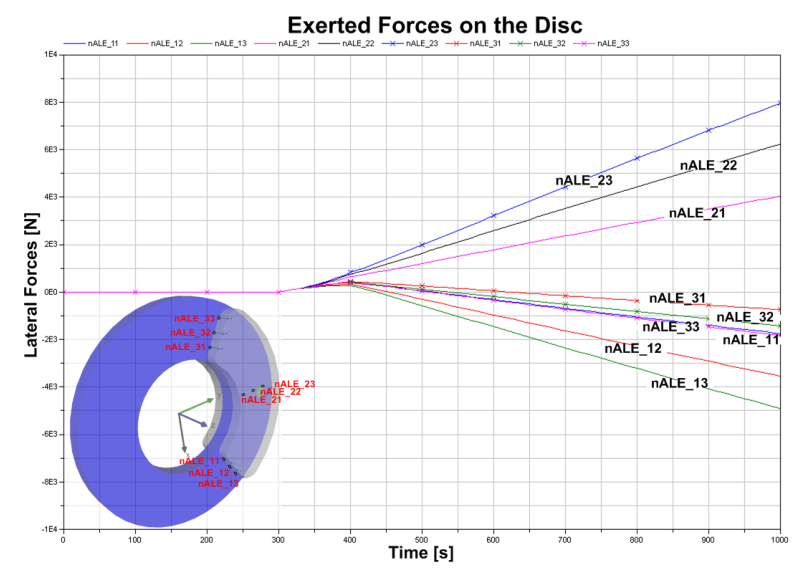

Figure 7: Results of the thermalPlate model for the normal forces acting on the ALE nodes.

As it was explained before the brake disc is rotating with a constant angular velocity. When the pads are in contact with the disc the kinetic energy is trans- formed into heat which results in an increase of the temperature in the disc. Figure 8 shows clearly the temperature propagation over the whole brake disc. The produced ring-shaped zone has a higher temperature than the rest of the disc due to the contact forces that the disc is exposed to.

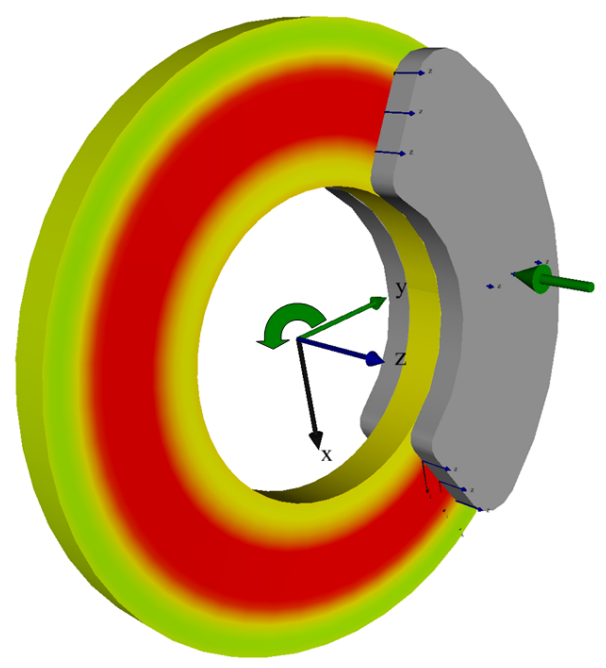

Figure 8: Heat propagation of the thermo-elastic plate with constant angular velocity.

It should be mentioned that these preliminary results still must be validated; nevertheless the results can be interpreted in a physical way and are plausible.

The example presented previously is the representation of a simplified brake disc model, including only braked disc and brake pads, but it represents the basis for a complete modeling of a braking mechanism.

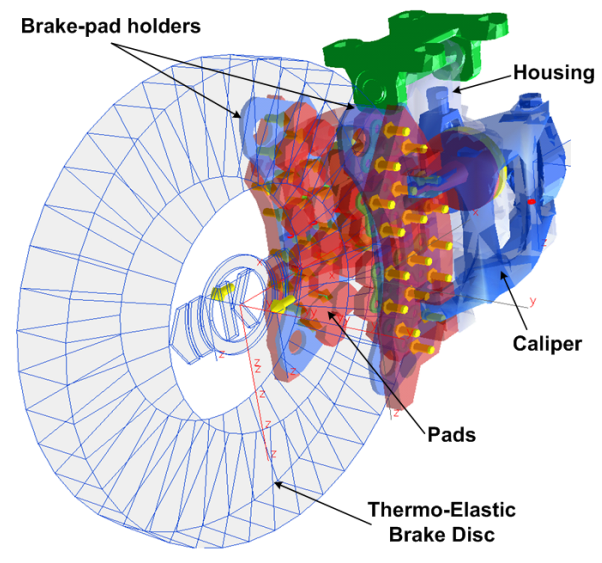

Figure 9: Braking mechanism of a train.

The purpose of this project is to integrate the thermo-elastic model into more complex scenarios, such as: complete braking system of a train (Figure 9 ) which includes brake disc (thermo-elastic model), 
brake pads, rockers, brake pad holders, calipers, housing, brake piston, etc., in order to analyze the induced vibrations, due to the thermo-mechanical deformations, into the complete dynamics of the entire system.

\section{Conclusion}

The present study investigates the thermo-mechanical effects on the dynamics of a simplified braking system by combining the FEM with the modal approach for flexible bodies in multibody systems. Validation of the presented analysis is still a pendent task; however the results have shown a good agreement with the physical description of this phenomenon giving a solid basis to cope with some of the most common braking problems such as hot spotting.

\section{Acknowledgements}

The authors highly appreciate the partial financial support of Knorr-Bremse Systems for Railway Vehicles in Munich.

\section{References}

[1] S. Panier, P. Dufrénoy, and D. Weichert. An experimental investigation of hot spots in railway disc brakes. Wear, 256:764 - 773, 2004.

[2] T.K. Kao, J.W. Richmond, and A. Douarre. Thermo-mechanical instability in braking and brake disc thermal judder: an experimental and finite element study. In Proc. of 2nd International Seminar on Automotive Braking, Recent Developments and Future Trends, IMechE, pages 231-263, Leeds, UK, 1998.

[3] A. Rinsdorf. Theoretische und experimentelle Untersuchungen zur Komfortoptimierung von Scheibenbremsen. Höppner und Göttert, Siegen, 1996.

[4] T. Steffen. Untersuchung der Hotspotbildung bei Pkw-Bremsscheiben. Number 345 in VDI-Fortschrittsberichte Reihe 12. VDI-Verlag, Düsseldorf, 1998.

[5] T. Tirovic and G.A. Sarwar. Design synthesis of non-symmetrically loaded high-performance disc brakes, Part 2: finite element modelling.
Proc. of the I Mech E Part F: Journal of Rail and Rapid Transit, 218:89 - 104, 2004.

[6] P. Dufrénoy. Two-/three-dimensional hybrid model of the thermomechanical behaviour of disc brakes. Proc. of the I Mech E Part F: Journal of Rail and Rapid Transit, 218:17 - 30, 2004.

[7] K. Lee and J.R. Barber. Frictionally excited thermoelastic instability in automotive disk brakes. Journal of Tribology, 115:607 - 614, 1993.

[8] C. Krempaszky and H. Lippmann. Frictionally excited thermoelastic instabilities of annular plates under thermal pre-stress. Journal of Tribilogy, 127:756-765, 2005.

[9] B.A. Boley and J.H. Weiner. Theory of Thermal Stresses. Dover Publications, Mineola, New York, 1997.

[10] H.J. Bathe. Finite Element Procedures. Prentice Hall, New Jersey, 1996.

[11] R.W. Lewis, K. Morgan, H.R. Thomas, and K.N. Seetharamua. The Finite Element Method in Heat Transfer Analysis. John Wiley and Sons, Chichester, UK, 1996.

[12] W. Ritz. Über eine neue Methode zur Lösung gewisser Variationsprobleme der mathematischen Physik. Journal für Reine und Angewandte Mathematik, 135:1-65, 1908.

[13] Carl de Boor. A practical Guide to Splines. Springer-Verlag, Berlin, 1978.

[14] A. Heckmann. The Modal Multifield Approach in Multibody Dynamics. Number 398 in FortschrittBerichte VDI Reihe 20. VDI-Verlag, Düsseldorf, 2005. PhD thesis.

[15] J. Salençon. Handbook of Continuum Mechanics. Springer-Verlag, Berlin, 2001.

[16] A. Heckmann, S. Hartweg, and I. Kaiser. An Annular Plate Model in Arbitrary LagrangianEulerian-Description for the DLR FlexibleBodies Library. In 8th International Modelica Conference, 2010. submitted for publication.

[17] O. Wallrapp and R. Schwertassek. Representation of geometric stiffening in multibody system simulation. International Journal for Numerical Methods in Engineering, 32:1833-1850, 1991. 
[18] F. Bloom and D. Coffin. Handbook of Thin Plate Buckling and Postbuckling. Chapman \& Hall/CRC, Washington, D.C., 2001.

[19] P.E. Nikravesh. Computer-aided Analysis of Mechanical Systems. Prentice Hall, Engelwood Cliffs, New Jersey, 1988. 